\title{
Prise en compte des savoirs paysans en matière de choix variétal dans un programme de sélection
}

\author{
Salifou Sissoko ${ }^{1}$ \\ Salif Doumbia ${ }^{2}$ \\ Michel Vaksmann ${ }^{3}$ \\ Henri Hocdé ${ }^{8}$ \\ Didier Bazile ${ }^{4}$ \\ Bougouna Sogoba ${ }^{5}$ \\ Mamoutou Kouressy ${ }^{1}$ \\ Kirsten Vom Brocke ${ }^{6}$ \\ Mamadou Mory Coulibaly ${ }^{1}$ \\ Aboubacar Touré 1 \\ Boureïma Gnalibouly Dicko ${ }^{7}$ \\ 1 Institut d'économie rurale (IER), Sotuba, BP \\ 438, Bamako, Mali \\ $<$ salifoube@yahoo.fr> \\ <Mamoutou.kouressy@ier.ml> \\ <madoumory@yahoo.fr> \\ <acar.btoure@ier.ml> \\ 2 Institut d'économie rurale (IER), BP 16, \\ Sikasso, Mali \\ <doumbiafsalif@yahoo.fr> \\ ${ }^{3}$ Centre de coopération internationale en \\ recherche agronomique pour le \\ développement (Cirad), BP 1813, Bamako, \\ Mali \\ <michel.vaksmann@cirad.fr>
}

${ }^{4}$ Cirad, Département Environnements \& Sociétés, UPR 47 " GREEN », Campus international de Baillarguet,

TA C-47/F,

34398 Montpellier cedex 5

<didier.bazile@cirad.fr>

${ }^{5}$ Association malienne d'éveil au développement durable (AMEDD), BP 212 , Koutiala, Mali

<bsogoba67@yahoo.fr>

${ }^{6}$ Cirad, 01 BP 596, Ouagadougou, Burkina Faso

<kirsten.vom_brocke@cirad.fr>

7 DELTA-C, BP E4850, Bamako, Mali <gnalibouly@yahoo.fr>

${ }^{8}$ Cirad, Département Environnements et sociétés, Unité de recherche (UR) " Actions collectives, politiques et marchés $", 73$, rue Jean-François Breton, 34398 Montpellier cedex 5

<henri.hocde@cirad.fr>

Tirés à part : M. Vaksmann

\begin{abstract}
Résumé
Un projet de sélection participative du mil a été initié pour comprendre les raisons de la faible diffusion des variétés améliorées dans la zone soudano-guinéenne du Mali. Un diagnostic et une prospection des variétés locales ont été réalisés en 2007 dans cinq villages de la région de Sikasso. Un essai comportant deux dates de semis (18 juin et 16 juillet 2007) a été réalisé à la station agricole de Sotuba. La durée du cycle de 29 variétés locales a été mesurée. Toutes les variétés prospectées sont photopériodiques, leur cycle diminue lorsque le semis est retardé. Les nouvelles variétés proposées aux paysans sont précoces, peu sensibles à la photopériode. Les chercheurs et les paysans sont convaincus que des variétés plus précoces que les cultivars locaux permettront d'éviter les sécheresses de fin de cycle. En réalité, les variétés locales résultent d'une sélection massale ancestrale et sont souvent spécifiquement adaptées à leurs environnements d'origines. La floraison des variétés locales est naturellement synchrone avec la date moyenne de fin de la saison des pluies. Ce caractère permet aux variétés locales de surmonter la principale incertitude climatique de la zone : la variabilité du début de la saison des pluies. La faible prise en compte des qualités propres aux variétés locales par les programmes d'amélioration des plantes explique le rejet des nouveaux cultivars par les paysans. Les programmes de sélection s'appuient maintenant sur le germoplasme local et un volet essentiel de l'expertise paysanne devient accessible à l'analyse des chercheurs.
\end{abstract}

Mots clés : amélioration des plantes, approches participatives, connaissance locale, photopériode, précocité.

Thèmes : productions végétales, ressources naturelles et environnement.

\section{Abstract \\ Accounting for farmer knowledge in varietal choice in a plant breeding programme}

A participatory plant breeding project has been initiated to understand the poor adoption of improved varieties of pearl millet in the soudano-Guinean zone of Mali. A prospection and a participatory diagnostic of cultivars was carried out in 5 villages of the Sikasso region in 2007. A trial comprised of two planting dates (18 June and 16 July 2007) was carried out at the agricultural station of Sotuba. The cycles of 29 local varieties were measured. All the tested varieties were photosensitive. Their cycles decreased with delayed planting. The new varieties proposed to the farmers are of the early-maturing type. The researchers and the farmers are convinced that early-maturing cultivars will make it possible to avoid terminal droughts. In fact, landraces originate from ancestral mass selection and are often well adapted to their local environments. Farmers selected photoperiodic varieties the flowering of which is naturally synchronous with the average date of the end of the rainy season. This trait allows pearl millet landraces to bypass the main climatic uncertainty of the zone: the variability of the beginning of the rainy season. The inadequate exploration of the qualities of local varieties by plant breeding programs can explain the rejection of new cultivars by farmers. Plant breeding programs nowadays put emphasis on local germoplasm, rendering farmers' knowledge and expertise perceivable to research analysis.

Key words: local knowledge, participatory approaches, plant breeding, photoperiod, precocity.

Subjects: natural resources and environment, vegetal productions. 
es premiers travaux d'amélioration du mil (Pennissetum glaucum) en Afrique de l'Ouest ont surtout porté sur la bande sahélienne. En 1999, les paysans de la zone cotonnière du Mali, à travers les Commissions d'utilisateurs des produits de la recherche, ont demandé à l'Institut d'économie rurale (IER) de lancer des recherches sur la mise au point de variétés de mils adaptées à la zone soudano-guinéenne (pluviométrie annuelle supérieure à $1000 \mathrm{~mm}$ ).

L'approche participative a été préconisée pour mieux impliquer les bénéficiaires du programme de sélection dans le processus d'identification et d'analyse des problèmes puis dans la recherche de solutions. La méthode d'analyse rapide et de planification participative (méthode MARP), très utilisée au Mali (Gueye et Freudenberger, 1991), a été choisie. Les préférences des paysans portaient sur la productivité, l'adaptation au climat et aux types de sol. Les paysans ont recensé les principales contraintes liées à la culture du mil dans leurs villages. Il est apparu que la principale cause d'abandon des variétés locales était l'inadaptation aux conditions climatiques actuelles (insuffisance de pluies). Les termes de référence d'un projet d'évaluation variétale participative ont été élaborés sur cette base et l'introduction chez les paysans de variétés plus précoces a été tentée en 2000 et 2001. L'utilisation de cultivars précoces a entraîné l'apparition de fortes contraintes biotiques (attaques d'oiseaux et moisissure du grain). Les nouvelles variétés se sont donc avérées peu performantes dans les systèmes de culture paysans et ont majoritairement été rejetées.

L'IER a donc décidé, en 2007, de compléter l'étude initiale par une caractérisation fine de la durée du cycle des variétés locales de la zone concernée. Il s'agissait d'initier un nouveau programme de création variétale devant déboucher sur un matériel correspondant aux pratiques et aux attentes des paysans de la zone soudano-guinéenne du Mali.

\section{Matériel et méthode}

\section{Enquêtes et prospections}

L'étude des variétés locales a été réalisée en 2007 dans les cinq villages où la tentative de vulgarisation de variétés précoces avait échoué. Ces villages couvrent les principales zones agricoles du sud de la

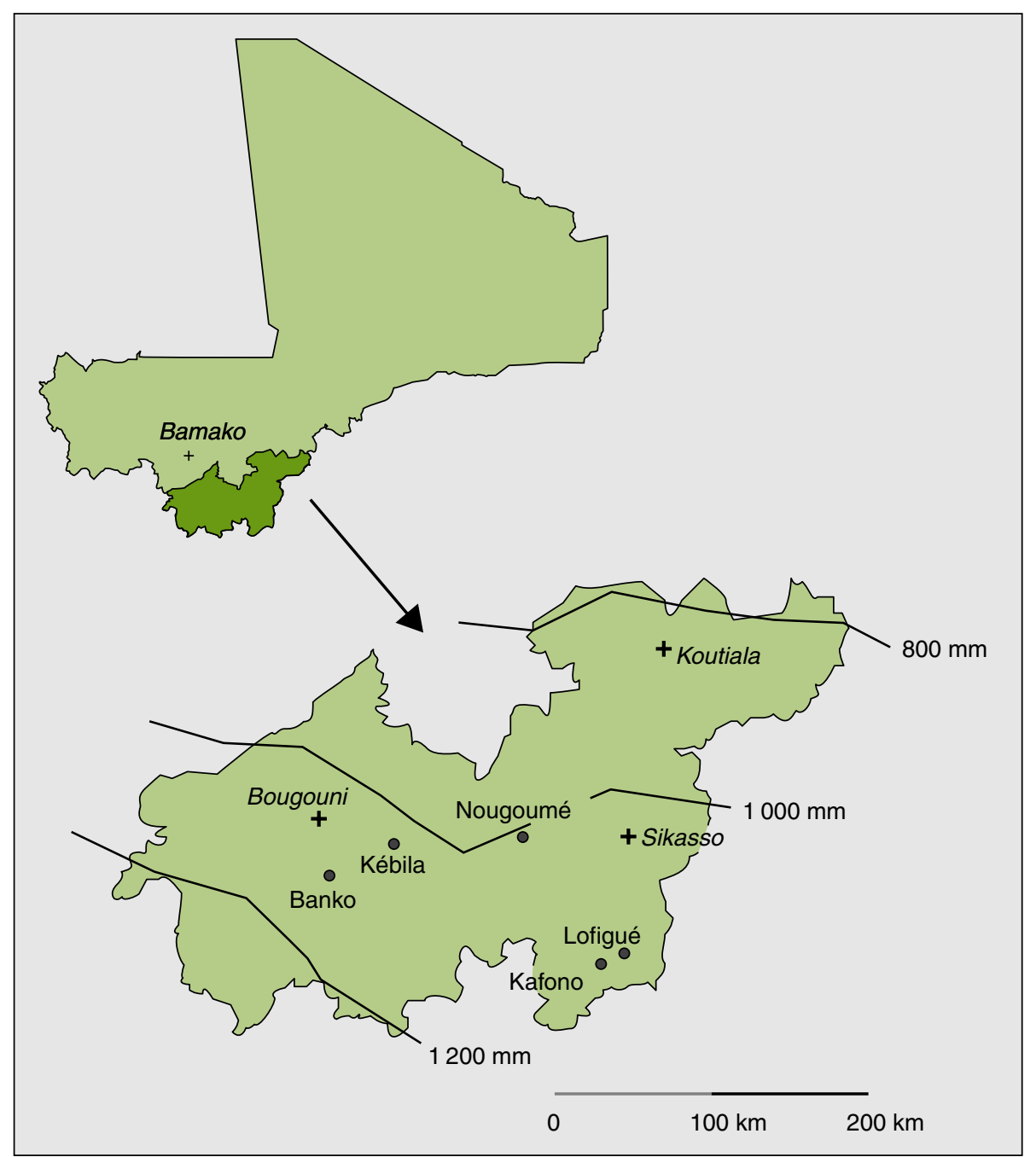

Figure 1. Région de Sikasso au Mali. Position des villages étudiées (Banko, Kébila, Nougoumé, Kafono, Lofigué) et pluviométrie moyenne sur la période 1971-2000.

Figure 1. Studied villages in the Sikasso region of Mali (circles) and average rainfall over the period 1971-2000.

région de Sikasso avec une pluviométrie supérieure à $1000 \mathrm{~mm}$ (figure 1). Un échantillonnage représentatif de la diversité des mils cultivés au sein de ces villages a été constitué sur la base d'entretiens semi-structurés en assemblées villageoises. Les questions étaient adressées à toute l'assemblée. Chaque paysan amenait un échantillon de ses variétés et des questions individuelles concernant le matériel fourni lui étaient adressées. Cette méthode de prospection permet d'accéder facilement à la diversité des mils d'un village. Son inconvénient est de donner un poids égal à toutes les variétés car les cultivars rares et fréquents sont considérés pareillement. Le nom vernaculaire des variétés n'a pas été retenu comme critère principal de choix : plusieurs échantillons pouvaient porter le même nom. Le nom- bre de variétés prospectées a été très variable selon les villages (tableau 1). Au total 29 variétés locales ont été obtenues.

\section{Caractérisation des variétés}

Le mil est une plante photopériodique de " jours courts ", sa floraison est accélérée lorsque la durée du jour se raccourcit. Comme la période de culture se déroule essentiellement après le 20 juin, en conditions de jours décroissants, le photopériodisme des mils provoque un raccourcissement du cycle lorsque la date de semis est retardée. Le cycle des 29 variétés a été mesuré à la station agricole de Sotuba ( $7^{\circ} 56^{\prime}$ Ouest et $12^{\circ} 39^{\prime}$ Nord) pour deux dates de semis : le 18 juin et le 16 juillet 2007. Le dispositif expérimental a été un split-plot variétés $\mathrm{x}$ dates de semis à 
Tableau 1. Durée semis-épiaison des variétés de la région de Sikasso pour le semis du 18 juin (SEP1) et du 16 juillet (SEP2) et coefficient de photopériodisme (Kp).

Table 1. The duration of the planting-heading period for cultivars planted in the Sikasso region on June 18, 2007 (SEP1) and July 16, 2007 (SEP2) and photosensitivity coefficients $(\mathrm{Kp})$.

\begin{tabular}{|c|c|c|c|c|c|c|}
\hline Numéro & $\begin{array}{c}\text { Nom de la } \\
\text { variété }\end{array}$ & Village & Latitude_ongitude & SEP1 & SEP2 & Kp \\
\hline 1 & Chô Wali & Lofigué & $10^{\circ} 38^{\prime} \mathrm{N} \quad 5^{\circ} 42^{\prime} \mathrm{O}$ & 121 & 97 & 0,86 \\
\hline 2 & Pan nègue & & & 125 & 99 & 0,91 \\
\hline 3 & Nioba & & & 127 & 102 & 0,89 \\
\hline 4 & Chô Wali & & & 125 & 101 & 0,84 \\
\hline 5 & Chô Wali & & & 126 & 98 & 1,00 \\
\hline 6 & Nioba & & & 127 & 98 & 1,00 \\
\hline 7 & Pan nègue & & & 124 & 99 & 0,89 \\
\hline 8 & Kon nagan & & & 126 & 98 & 1,00 \\
\hline 9 & Chô Wali & & & 117 & 94 & 0,80 \\
\hline 10 & Kon nagan & Kafono & $10^{\circ} 35^{\prime} \mathrm{N} 5^{\circ} 50^{\prime} \mathrm{O}$ & 118 & 96 & 0,80 \\
\hline 11 & Chô Wali & & & 119 & 96 & 0,82 \\
\hline 12 & Kon nagan & & & 121 & 94 & 0,96 \\
\hline 13 & Chô Wali & & & 119 & 93 & 0,95 \\
\hline 14 & Womèrè nagan & & & 118 & 93 & 0,91 \\
\hline 15 & Womèrè nagan & & & 125 & 99 & 0,93 \\
\hline 16 & Kon nagan & & & 124 & 95 & 1,00 \\
\hline 17 & Kon nagan & & & 125 & 96 & 1,00 \\
\hline 18 & Kon nagan & & & 125 & 97 & 1,00 \\
\hline 19 & Womèrè nagan & & & 124 & 96 & 0,98 \\
\hline 20 & Chô Wali & & & 119 & 92 & 0,96 \\
\hline 21 & Sanio Koumaba & Nougoumé & $11^{\circ} 19^{\prime} \mathrm{N} 6^{\circ} 17^{\prime} \mathrm{O}$ & 99 & 79 & 0,70 \\
\hline 22 & $\begin{array}{l}\text { Sanio } \\
\text { Souroman }\end{array}$ & & & 99 & 75 & 0,86 \\
\hline 23 & $\begin{array}{l}\text { Sanio } \\
\text { Misséman }\end{array}$ & & & 100 & 80 & 0,70 \\
\hline 24 & Sanioba & Banko & $11^{\circ} 6^{\prime} \mathrm{N} \quad 7^{\circ} 25^{\prime} \mathrm{O}$ & 105 & 82 & 0,84 \\
\hline 25 & Sanioba & & & 102 & 81 & 0,75 \\
\hline 26 & Nioba & Kebila & $11^{\circ} 17^{\prime} \mathrm{N} 7^{\circ} 02^{\prime} \mathrm{O}$ & 104 & 81 & 0,82 \\
\hline 27 & Flanio & & & 103 & 81 & 0,77 \\
\hline 28 & Laiguè & & & 112 & 88 & 0,86 \\
\hline \multirow[t]{2}{*}{29} & Flanio & & & 106 & 80 & 0,91 \\
\hline & Guéfoué 16 & \multicolumn{2}{|c|}{ Exemple de variété vulgarisée } & 80 & 69 & 0,39 \\
\hline
\end{tabular}

2 répétitions. Le photopériodisme des variétés a été évalué à partir du coefficient $K p$ qui mesure le raccourcissement de la période végétative lorsque le semis est retardé. Ce coefficient est défini par le rapport de la différence des durées semisépiaison entre la première et la seconde date de semis (SEP1 - SEP2) sur le nombre de jours $(\Delta S)$ entre les 2 semis (Traoré et al., 2000). Il varie de 0 à 1 suivant le photopériodisme des variétés.

$$
K p=\frac{S E P 1-S E P 2}{\Delta S}
$$

ment bien adaptées aux conditions de cultures traditionnelles. La variété idéale décrite par les paysans ressemble beaucoup à leurs propres variétés même s'il faudrait la rendre plus productive, précoce et insensible à la moisissure et aux oiseaux. On peut relever ici une réelle contradiction entre les critères d'amélioration avancés puisque les moisissures et les attaques d'oiseaux résultent de l'augmentation de précocité.

\section{Durée du cycle des variétés locales}

L'étude conduite en station montre que les variétés locales sont très photopériodiques. La durée du cycle des variétés de la région de Sikasso diminue en moyenne de 25 jours lorsque le semis est retardé de 28 jours, ce qui correspond à un coefficient $K p$ de 0,89 (tableau 1). On observe deux groupes de précocités différentes. Pour le semis du 18 juin, les variétés des villages de Lofigué et Kafono épient en moyenne le 16 octobre, 120 jours après le semis, tandis que les variétés des trois autres villages, situés plus au nord, épient après 95 jours vers le 21 septembre.

Cette différence du cycle des variétés entre les villages s'explique plus par des différences de pratiques que par l'effet des contraintes climatiques. Dans tous les villages étudiés, les dates moyennes de début et de fin des pluies sont identiques, respectivement le 25 mai et le 11 octobre. Les variétés des villages du sud sont plus tardives. Elles épient souvent après les pluies et le grossissement du grain se fait essentiellement sur l'eau stockée dans le sol. Ces variétés témoignent probablement d'anciennes pratiques culturales, à présent marginalisées, comme la culture associée maïs-mil (Niangado, 1989). Le mil est semé ou repiqué directement 15 à 20 jours après le maïs et se développe surtout après la récolte du maïs. Cette association de cultures nécessite des variétés de mils particulièrement tardives pour diminuer la compétition entre les deux céréales dont les cycles se chevauchent.

\section{Enquêtes}

Les facteurs limitant la production du mil, exprimés par les paysans au cours des nouvelles enquêtes ne diffèrent pas beaucoup de ceux relevés dans le diagnostic participatif initial. L'irrégularité des pluies et la pauvreté des sols constituent les principaux problèmes rencontrés. En revanche, les paysans semblent relativement satisfaits de leurs variétés qu'ils esti-
En comparaison, les variétés améliorées proposées par la recherche sont beaucoup plus précoces que toutes les variétés locales (tableau 1).

Nous n'avons pas trouvé dans notre échantillonnage de variétés précoces pouvant être utilisées pour faire face à la période de disette fréquente avant la récolte principale de l'année (période de soudure). Le maïs, plus résistant à l'excès 
d'eau au moment de la maturité du grain, est généralement préféré pour cet usage. Il peut être consommé en octobre et novembre avant les récoltes de mils et sorghos. Le maïs s'est aussi développé récemment à proximité des villes car cette culture bénéficie maintenant de débouchés sur les marchés en "maïs de bouche "(épis grillés).

\section{Discussion}

\section{Incertitude climatique et date de semis}

La durée de la saison des pluies détermine le potentiel de production agricole. C'est surtout le début des pluies qui est très variable d'une année à l'autre (figure 2) conduisant à une très forte variabilité interannuelle de la durée de la saison des pluies (Sivakumar, 1988).

À cette variabilité de l'arrivée des pluies se surimpose un étalement important des semis. Le début de saison est toujours une course de vitesse pour les paysans du Mali car le rendement des céréales est maximisé si les semis sont réalisés tôt avec les premières pluies substantielles. Les semis précoces améliorent la maîtrise de l'enherbement et facilitent l'installation de la culture. Mais toutes les parcelles sont rarement semées simultanément. Des précipitations erratiques en début de saison entraînent la succession de plusieurs vagues de semis. Il n'est pas rare que les paysans ressèment deux ou trois fois, soit à cause de mauvaises levées, soit à cause d'attaques de déprédateurs. De plus, les contraintes d'exploitation, le manque de main-d'œuvre ou de matériel agricole ne permettent pas aux paysans de semer rapidement l'ensemble de leurs champs. Les semis débutent au moment de l'installation des pluies et s'étalent généralement sur plus d'un mois.

\section{Le photopériodisme : caractère d'adaptation des variétés locales}

Pour tenir compte de la variabilité du climat, les agriculteurs ont sélectionné des céréales photopériodiques spécifiquement adaptées à leur environnement très changeant. Les paysans définissent les écotypes adaptés par "ils s'attendent pour mûrir ensemble ", quelles que soient leurs dates de semis ( $u$ be nyogon konô).

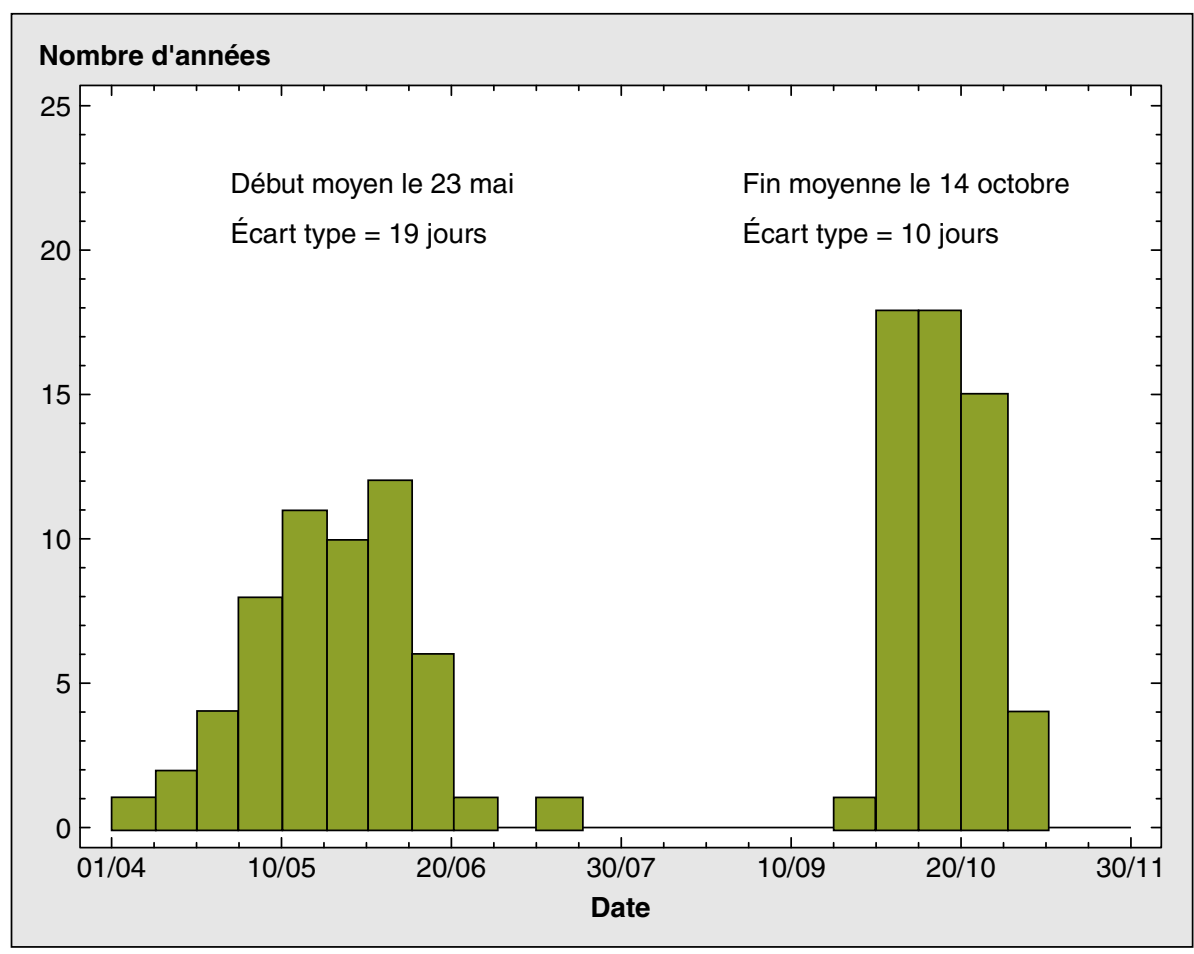

Figure 2. Distribution des dates de début et de fin de saison des pluies à Bougouni (1950-2005).

Figure 2. Distribution of starting and cessation dates of rains in Bougouni (1950-2005).

Le début de saison qui détermine les premières vagues de semis est très variable d'une année sur l'autre.
Le photopériodisme des variétés locales permet la synchronisation de la floraison avec la fin de la saison des pluies. Une variété peut être considérée comme adaptée à une zone si la floraison débute dans les 20 jours qui précèdent la fin moyenne de la saison (Traoré et al., 2007). Cette condition assure un équilibre entre la satisfaction des besoins en eau et l'évitement de nombreuses contraintes biotiques. Le rendement et la qualité du grain dépendent étroitement de la date de floraison car le grain des variétés qui fleurissent trop tôt est attaqué par les oiseaux et altéré par les moisissures et les insectes. Les variétés dont la floraison est trop tardive épuisent les réserves en eau du sol avant la fin du remplissage des grains.

Le photopériodisme des variétés locales de mil et de sorgho confère à ces deux espèces une place privilégiée dans les systèmes de culture à base de coton et de maïs car le paysan peut facilement étaler ses semis de mils et de sorghos pour adapter la gestion de sa main-d'œuvre aux besoins des différentes spéculations de son assolement.

\section{Le besoin de variétés précoces : mythe ou réalité ?}

La recherche de plantes précoces est un postulat fondamental de la révolution verte (Swaminathan, 2006) et l'élimination du photopériodisme est devenue un préalable à la plupart des programmes d'amélioration des céréales. Les sécheresses des années 1970 dans le Sahel ont conforté cette position. L'élimination du photopériodisme devenait une nécessité pour aboutir à un matériel précoce capable de supporter des saisons des pluies de plus en plus courtes (Dancette, 1983). Le discours ambiant fait que les paysans sont aussi devenus convaincus de l'intérêt de la précocité, en zone sèche comme en zone humide. Le mot "tardif " est même devenu péjoratif, c'est pourquoi les variétés qui plaisent aux paysans sont parfois dites "précoces " indépendamment de la durée réelle de leur cycle. Pour cette raison, au cours des diagnostics participatifs, la précocité apparaît toujours comme le principal caractère demandé par les paysans (Omanya et al., 2007). Il faudrait certainement clarifier le sens réel que les paysans donnent à ce terme dans chaque environnement.

Cette convergence apparente entre les croyances des chercheurs et des paysans 
a débouché sur la création de nombreuses variétés de cycles courts qui se sont avérées inadaptées. En effet, les contraintes climatiques justifient rarement l'adoption de variétés plus précoces que les cultivars locaux. La baisse de pluviométrie observée dans la zone soudanosahélienne depuis 40 ans ne s'est pas accompagnée d'une modification importante de la durée de la saison des pluies (Barbe et Lebel, 1997; Traoré et al., 2000). La sécheresse a donc eu peu de conséquences sur la longueur des cycles des plantes de culture pluviale. La majeure partie de l'échec à la diffusion des variétés améliorées peut s'expliquer par leur trop grande précocité qui oblige les paysans à retarder le semis après le démarrage de la saison. C'est une contrainte majeure pour la gestion du travail au sein de l'exploitation agricole. Des résultats similaires ont été obtenus en zone sahélienne où les variétés améliorées de cycles courts ont été refusées par les paysans au profit des variétés traditionnelles mieux adaptées au climat et aux pratiques de semis très précoces de la zone (de Rouw, 2004).

\section{Prise en compte des savoirs paysans en matière de choix variétal}

La définition des objectifs de sélection et le choix des géniteurs sont deux préalables essentiels à la mise en place d'un programme d'amélioration variétale. La définition participative des variétés à créer est souvent délicate car les paysans éprouvent des difficultés à construire des idéotypes nouveaux. Leur expérience est limitée à une zone restreinte (village ou petite région naturelle) et ils n'ont pas connaissance de l'étendue de la diversité génétique disponible.

Les paysans (comme d'ailleurs la plupart des agronomes) remarquent en premier lieu les imperfections du matériel. Ils éliminent une variété sur la base d'un seul défaut. Cette démarche est justifiée pour une évaluation variétale visant à retenir le matériel cultivable en l'état. En revanche, en phase de création de nouvelles variétés, l'élimination trop rapide d'une descendance entraîne une baisse de variabilité préjudiciable au progrès génétique à long terme. C'est pourquoi l'implication des paysans ne peut pas être égale pendant tout le processus d'amélioration participative. Dans la phase de création variétale, les paysans orientent la sélection par leurs pratiques (travail du sol, choix des densités et dates de semis, gestion de la fertilité). Leur rôle s'accrôtt ensuite, en phase d'évaluation, lorsque le matériel est suffisamment homogène pour être considéré comme une variété qu'ils pourront plus aisément juger.

Le savoir paysan peut être défini comme étant l'ensemble des expériences et connaissances qu'un groupe ethnique utilise dans le processus de prise de décision concernant les problèmes et défis qui l'interpellent (Warren et Cashman, 1988). Les variétés locales ont été élaborées au cours des siècles et se transmettent de générations en générations. Elles incorporent une partie de savoirs et de pratiques qui sont souvent difficiles à percevoir de l'extérieur car les paysans ne cherchent pas à les justifier dans leurs discours. Pour s'assurer de l'adaptation au milieu des nouvelles créations variétales il semble approprié d'intégrer les cultivars locaux des zones agroclimatiques cibles.

\section{Adaptation au sol ou adaptation au climat}

En matière d'amélioration des céréales, la démarche participative cherche fréquemment à créer des variétés capables de surpasser les écotypes locaux dans des conditions marginales, de faible fertilité (Bänziger et Cooper, 2001; Sperling et al., 2001). Pourtant les nouvelles variétés proposées par la recherche en Afrique de l'Ouest ne sont pas plus productives que les cultivars locaux des paysans (Omanya et al., 2007; Ahmed etal., 2000). Au Mali, les tests variétaux montrent même souvent le contraire avec une supériorité des céréales locales par rapport au matériel amélioré, que ce soit en stations de recherche (Luce, 1994) ou en champs paysans (Lacy et al., 2006).

Chercher à surpasser les variétés locales dans leurs propres systèmes traditionnels extensifs s'avère donc un objectif de sélection très difficile à réaliser. Les paysans disposent déjà d'un ensemble très vaste de variétés capables de produire sur des sols peu fertiles et sous des climats incertains. En revanche, dans des conditions de plus forte productivité où l'environnement physique est moins contraignant (abandon de la culture itinérante, apports d'intrants, mécanisation), les variétés traditionnelles de mils n'arrivent plus à atteindre les objectifs de production que se fixent désormais les paysans. C'est pourquoi, dans la zone cotonnière, on observe actuellement une extension rapide du maïs qui répond mieux à l'intensification agricole (Bazile et Soumaré, 2004).

Traditionnellement, les paysans évitent de semer le mil sur des sols trop fertiles car la production de tiges est alors favorisée au détriment de celle du grain. L'amélioration de l'indice de récolte des variétés locales (ratio entre la biomasse récoltée et la biomasse aérienne totale) devrait, en partie, résoudre ce problème. Pour répondre aux nouvelles attentes des paysans des zones cotonnières d'Afrique de l'Ouest, le véritable défi à relever en termes d'amélioration variétale du mil serait de remonter la productivité des variétés locales, naturellement adaptées au climat, dans les systèmes de cultures en voies d'intensification.

\section{Conclusion}

Les savoirs locaux des agriculteurs sont le fruit d'un long processus d'adaptation aux conditions environnementales et socio-économiques. Ce processus ne fait pas l'objet d'une formalisation claire de la part des paysans qui connaissent empiriquement l'efficacité des techniques héritées de leurs parents. En s'appuyant sur les variétés locales, un volet essentiel de l'expertise paysanne devient accessible à l'analyse du chercheur. La prospection et la caractérisation de la diversité génétique doivent devenir des éléments clés des diagnostics participatifs. C'est le cas de l'adaptation de variétés de mil au climat soudano-guinéen. Les sécheresses des années 1970 et 1980 n'ont pas entraîné de changements profonds de la longueur de la saison des pluies et la plupart des variétés locales sont toujours adaptées à leurs milieux d'origines. C'est pourquoi les paysans ont sélectionné des variétés photopériodiques dont la floraison est naturellement synchrone avec la date moyenne de fin de la saison des pluies. Ce caractère permet aux variétés locales de surmonter la principale incertitude climatique de la zone: la variabilité du début de la saison des pluies.

Le programme malien d'amélioration du mil intègre maintenant une large base génétique locale et s'inspire du comportement des variétés locales de chaque milieu cible. Le matériel produit devra cumuler la souplesse des variétés locales et la productivité du matériel moderne. 


\section{Références}

Ahmed MM, Sanders JH, Nell WT. New sorghum and millet cultivar introduction in SubSaharan Africa : impacts and research agenda. Agric Sys $2000 ; 64$ : 55-65.

Bänziger M, Cooper M. Breeding for low input conditions and consequences for participatory plant breeding examples from tropical maize and wheat. Euphytica $2001 ; 122$ : 503-19.

Barbe LL, Lebel T. Rainfall climatology of the HAPEX-Sahel region during the years 1950-1990. J hydrol $1997 ;(188-189)$ : 43-73.

Bazile D, Soumaré M. Gestion spatiale de la diversité variétale en réponse à la diversite écosystémique: le cas du sorgho [Sorghum bicolor (L) Moench] au Mali. Cah Agric 2004 ; $13: 480-7$.

Dancette C. Besoins en eau du mil au Sénégal. Adaptation en zone semi-aride tropicale. Agron Trop 1983 ; 38 : 267-80. de Rouw A. Improving yields and reducing risks in pearl millet farming in the African Sahel. Agric Sys 2004 ; 81 : 73-93.

Gueye B, Freudenberger KS. Introduction à la méthode accélérée de recherche participative (MARP). London: International Institute for Environment and Development (IIED), 1991.

Lacy SM, Cleveland DA, Soleri D. Farmer choice of sorghum varieties in southern Mali. Hum Ecol 2006 ; 34 : 331-53.

Luce C. Le rendement d'écotypes de sorgho des races caudatum et guinea. Agriculture et Développement $1994 ; 2: 35-8$.

Niangado O. Production et amélioration variétale du mil au Mali. Plantes vivrières tropicales. Paris : Aupelf-Uref ; John Libbey Eurotext 1989.

Omanya GO, Weltzien-Rattunde E, Sogodogo $D$, et al. Participatory varietal selection with improved pearl millet in west africa. Exp Agric $2007 ; 43: 5-19$.
Sivakumar MVK. Predicting rainy season potential from the onset of rains in southern Sahelian and Sudanian climatic zones of West Africa. Agric For Meteorol 1988 ; 42 : 295-305.

Sperling L, Ashby JA, Smith ME, Weltzien E, McGuire S. A framework for analyzing participatory plant breeding approaches and results. Euphytica $2001 ; 122$ : 439-50.

Swaminathan MS. An evergreen revolution. Crop Sci 2006 ; 46 : 2293-303.

Traoré PCS, Kouressy M, Vaksmann M, et al. Climate prediction and agriculture: What is different about Sudano-Sahelan West Africa? In : Sivakumar MVK, Hansen J, eds. Climate prediction and agriculture. Berlin; Heidelberg : Springer Verlag, 2007.

Traoré SB, Reyniers F-N, Vaksmann M, et al. Adaptation à la sécheresse des écotypes locaux de sorghos du Mali. Sécheresse 2000 ; $11: 227-37$.

Warren DM, Cashman K. Indigenous knowledge for sustainable agriculture and rural development. London : International Institute for Environment and Development (IIED), 1988. 